\title{
Fortbildung und Fortbildungskontrolle: Der Standpunkt der SGIM
}

\author{
Ruedi Frey, Präsident der Fortbildungskommission SGIM
}

Die SGIM, die grösste Fachgesellschaft der Schweiz, ist im Rahmen des Fortbildungsobligatoriums verpflichtet, Fortbildungskontrollen durchzuführen. Die SGIM tut auf dem Gebiet der Fortbildung aber mehr, als überlastete Ärzte mit Stichproben zu ärgern. Die SGIM bietet Fortbildungsveranstaltungen an, evaluiert die unterschiedlichsten Fortbildungsangebote und informiert über die anerkannten Veranstaltungen.

Dass sich Ärztinnen und Ärzte nach der Aus- und Weiterbildung während der ganzen beruflichen Laufbahn fortbilden, ist eine Selbstverständlichkeit. In den letzten Jahren hat sich auf dem Gebiet der Fortbildung aber einiges geändert: Die Fortbildung wurde obligatorisch und gesetzlich verankert. Dies bedeutet für den einzelnen Arzt, dass er seine Fortbildung nachweisen muss.

Gemäss Fortbildungsordnung der FMH haben die Fachgesellschaften den Auftrag, die für ihr Fachgebiet nötige Fortbildung zu definieren und die nötigen Kontrollen durchzuführen (im Minimum Stichproben). Wenn jemand die Richtlinien nicht einhält, die von der FMH und den Fachgesellschaften ausgearbeitet wurden, sind (noch) keine Sanktionen vorgesehen. Das heisst aber nicht, dass ein Arzt, der seiner Fortbildungspflicht nicht nachkommt, gar nichts zu fürchten hat. Probleme könnten zum Beispiel auftauchen bei Streitigkeiten zwischen Arzt und Patient oder zwischen Arzt und Versicherung.

\section{Die SGIM hat eine klare Linie}

Auch die SGIM ist verpflichtet, die Fortbildung in ihrem Fach zu definieren (Fortbildungsprogramm) und Kontrollen durchzuführen. Die SGIM hat ihre Aufgaben gemacht und ein Konzept für die Fortbildungskontrolle erarbeitet. Man hört in der SGIM aber auch immer wieder den lautstarken Ruf der Internistinnen und Internisten nach möglichst freiheitlicher Gestaltung der Fortbildung. Wie geht die SGIM mit dieser Situation um?

Alle zufriedenzustellen, ist ein Ding der UnKorrespondenz: SGIM ten permanent und optimal fortbilden - nach wissenschaftlichen, ethischen und wirtschaftlichen Kriterien. Dabei ist der SGIM vor allem die Qualität des Fortbildungsangebots wichtig.

\section{Das Fortbildungsprogramm der SGIM}

Das Fortbildungsprogramm der SGIM ist inhaltlich so aufgebaut, dass die Internisten in Spital und Praxis mit der Fortbildung ihre medizinischen «Grundbedürfnisse» decken können. Es geht dabei um Innere Medizin. Dieser Begriff umfasst im Fortbildungsprogramm alles, was ein «durchschnittlicher» Internist in seinem Beruf braucht. Nicht dazu gehören Gebiete, die eindeutig ausserhalb der Inneren Medizin anzusiedeln sind (zum Beispiel Alternativmedizin) oder administrative und andere Fächer (zum Beispiel TARMED).

Es ist die Meinung der SGIM, dass eine Fachgesellschaft für die Fortbildung in denjenigen Wissensgebieten verantwortlich ist, die zum Fachgebiet der Gesellschaft gehören. Eine Fachgesellschaft ist aber nicht zuständig für die Fortbildung des gesamten Wissens, das ein Arzt kontinuierlich braucht und aktualisieren muss, zum Beispiel EDV-Kenntnisse oder Versicherungsfragen.

Wie der Bedarf an jährlicher Fortbildung in einem Fach errechnet werden kann, ist eine offene Frage. Die aktuell gültigen und obligatorischen 50 Stunden pro Jahr (150 Stunden pro Dreijahresperiode) sind vermutlich noch nicht der Weisheit letzter Schluss.

\section{Die Fortbildungsveranstaltungen der SGIM}

Die Fachgesellschaften haben auch die wichtige Aufgabe, Fortbildungsveranstaltungen anzubieten. Die SGIM ist auf diesem Gebiet sehr aktiv. Der jährliche SGIM-Kongress hat sich in den letzten Jahren von einer etwas elitären, spitallastigen zu einer modernen, topaktuellen Veranstaltung gewandelt. Inzwischen ist der SGIMKongress der grösste medizinische Kongress in der Schweiz. 
Ausser dem Jahreskongress bietet die SGIM zwei verschiedene Formen von Kursen an. Die regionalen Kurse werden von der SGIM in eigener Regie organisiert, vorwiegend in wechselnden internmedizinischen A-Kliniken (2003 in Langenthal, Lausanne und Neuchâtel). Die regionalen Kurse erfreuen sich zunehmender Beliebtheit. Die SGIM beteiligt sich ausserdem an bereits bestehenden Kursen wie Davoser Kurs, Quadrimed, Fortbildungskurs der medizinischen Fakultät Zürich, Zentralschweizer Internistenkurs und Lenker Kurs. Bei der Organisation dieser Kurse wirkt ein Mitglied der Fortbildungskommission oder des Vorstands der SGIM mit.

Bei der SGIM besteht zudem ein Konzept für eine neue Kursform mit dem Titel «SGIM goes regional». Diese halbtägigen Kurse haben zum Ziel, die SGIM in den Regionen bekanntzumachen und zu verankern.

\section{Evaluation und Anerkennung von Fortbildungsveranstaltungen}

Die SGIM evaluiert die verschiedenen Fortbildungsangebote. Dabei werden nicht nur die Inhalte beurteilt, die gemäss Fortbildungsprogramm aus der Inneren Medizin bestehen müssen, sondern auch die Qualität einer Veranstaltung und die Beeinflussung der Inhalte von aussen (beispielsweise durch die Pharmaindustrie). Diese Beurteilung ist aus verschiedenen Gründen oft sehr komplex:

- Veranstaltungen müssen aufgrund der eingereichten Programme evaluiert werden und nicht aufgrund des persönlichen Eindrucks bei einer Teilnahme.

- Bei der Beurteilung muss die SGIM den verschiedenen Arten von Fortbildung gerecht werden, seien dies E-Learning, Engagement in der Lehre, Verfassen von wissenschaftlichen Arbeiten, Fortbildung in Qualitätszirkeln usw.

- Ohne Zusammenarbeit zwischen Ärztegesellschaften und Pharmaindustrie könnten nur noch wenig Fortbildungsangebote realisiert werden - dies ist eine Realität. Gerade deshalb gestaltet sich die Beurteilung von gesponserten Veranstaltungen oft besonders heikel.

Die Kriterien der SGIM zur Beurteilung von Fortbildungsveranstaltungen und zur Vergabe von Credits werden laufend verfeinert. Die bisherigen Erfahrungen zeigen, dass die Veranstalter an einer Validierung durch die SGIM sehr interessiert sind und dass die Qualitätskriterien der SGIM die Veranstalter positiv beeinflussen können.
Auf der SGIM-Website wurde kürzlich eine Datenbank aufgeschaltet, in der von der SGIM akkreditierte Fortbildungsveranstaltungen eingesehen werden können. Im Moment enthält die Datenbank über 1000 Fortbildungsangebote, die Zahl wächst rasch.

\section{Die SGIM gestaltet Entscheidungen mit}

Die SGIM versucht, die Diskussionen und Entscheidungen in den übergeordneten Gremien (Gesetzgeber, FMH usw.) mitzugestalten. Dabei vertritt die SGIM den Standpunkt, dass die Fortbildungsordnung dem Ziel einer qualitativ hochstehenden, freiheitlichen Medizin dient und keine weiteren Einschränkungen nach sich zieht.

\section{Fortbildungsnachweis entspricht dem Zeitgeist}

Das Fortbildungsobligatorium ist heftig umstritten. Spontane Reaktionen zu diesem Thema lauten häufig:

- «Bringt doch nichts.»

- «Die Ärzte haben sich bis jetzt auch fortgebildet.»

- «Die Verweigerer werden einen Weg finden, sich zu drücken.»

- «Wenn man all die Schlafenden in den Hörsälen sieht und die vielen 〈Teilnehmer〉, die nur bei der Testatverteilung anwesend sind, wird's fragwürdig.»

- «Niemand hat bewiesen, dass eine obligatorische Fortbildung auch die praktizierte Medizin und vor allem die Gesundheit der Patienten verbessert.»

Alles wahr. Und doch ist die Sache um einiges komplizierter und komplexer. Der Nachweis der Fortbildung wird von der Öffentlichkeit zunehmend gefordert. Die Haltung der Ärzteschaft, «Wir machen es schon richtig, ihr müsst uns nur vertrauen», verfängt nicht mehr. Gesetzgeber, Konsumentinnen- und Patientenorganisationen sowie Versicherer verlangen den Nachweis der absolvierten Fortbildung nach dem Motto «Vertrauen ist gut, Kontrolle ist besser». Die Tendenz der Bevölkerung und der Behörden, mehr Transparenz zu fordern und eine kritische Haltung einzunehmen, trifft nicht nur Ärztinnen und Ärzte, sondern auch andere Berufsgruppen. 


\section{Die SGIM und die Fortbildungskontrollen}

Gegenüber den Forderungen auf dem Gebiet der Fortbildung nimmt die SGIM eine konsequente Haltung ein. Da die Fortbildungskontrolle vorgeschrieben ist, macht es keinen Sinn, diese Kontrolle zur Alibiübung verkommen zu lassen. Die SGIM übt sich keineswegs in vorauseilendem und devotem Gehorsam gegenüber Gesetzgeber und Versicherern, die SGIM will aber auch vermeiden, dass die beschlossenen Stichproben zur Farce werden.

Die Erfahrungen der SGIM zeigen, dass sich die Mehrheit der Ärzte gewissenhaft und mit Engagement fortbildet. Auffallend ist aber auch, dass die Besucherzahlen an Fortbildungsveranstaltungen in die Höhe geschnellt sind, seit das Obligatorium eingeführt wurde. Auch die Zahl der Fortbildungsangebote hat deutlich zugenommen. Es stellt sich natürlich die Frage, wo sich all die «neuen» Besucher fortbildeten, bevor das Obligatorium existierte. Die Fortbildungsverantwortlichen der SGIM halten es aufgrund eigener Beobachtungen für eine Tatsache, dass sich die Qualität vieler Fortbildungsangebote seit Einführung des Obligatoriums verbessert hat.

Für eine Fachgesellschaft ist es oft schwierig, die berechtigten Forderungen ihrer Mitglieder und die Ansprüche von Behörden und Gesetzgebern unter einen Hut zu bekommen. Die SGIM bemüht sich auf dem Gebiet der Fortbildung um sinnvolle Lösungen. Die Verantwortlichen der SGIM sind sich bewusst, dass die SGIM als grösste Fachgesellschaft der Schweiz hier auch Schrittmacherfunktion ausübt.

\title{
La formation continue et son contrôle: le point de vue de la SSMI
}

\author{
Ruedi Frey, Président de la Commission pour la formation continue de la Société Suisse de Médecine Interne
}

Dans le cadre du devoir de formation continue, la SSMI, la plus grande société de discipline médicale suisse, est tenue d'effectuer des vérifications de la formation continue. Dans ce domaine, le rôle de la SSMI ne consiste pas simplement à importuner des médecins, déjà suffisamment débordés de travail, par des contrôles inopinés. La SSMI organise en effet des sessions de formation continue, procède à une évaluation de divers programmes de formation continue et propose des informations sur les manifestations agréées.

Il est tout à fait naturel que les médecins continuent à se perfectionner au cours de leur carrière, après avoir terminé leurs études et effectué leur formation postgraduée. Toutefois, ces dernières années, les choses ont passablement changé dans le domaine de la formation continue: celleci est devenue obligatoire, prescrite par la loi. Cela a pour conséquence que le médecin est désormais obligé de prouver qu'il a bien accompli la formation continue qu'il est censé effectuer chaque année.

Selon la Réglementation pour la formation continue de la FMH, les sociétés de discipline médicale sont chargées de définir la formation continue qu'elles estiment nécessaire dans leur spécialité, et également d'effectuer les contrôles appropriés (consistant au minimum en vérifications par sondage). Les médecins qui ne se conforment pas à la procédure élaborée par la FMH et les sociétés de discipline médicale, n'encourent pas (encore) de sanctions. Cela ne signifie pas pour autant qu'un médecin qui ne se plie pas aux exigences de la formation continue obligatoire n'a absolument rien à craindre. Il pourrait en effet rencontrer des problèmes en cas de litiges entre médecin et patient ou entre médecin et compagnie d'assurances.

\section{La SSMI a une ligne de conduite bien définie}

La SSMI est, elle aussi, chargée de définir la formation continue adéquate dans son domaine (Programme de formation continue) et de procéder à son contrôle. La SSMI s'est acquittée de ce devoir en élaborant un plan cadre pour le contrôle de la formation continue. Il arrive cependant que des internistes réclament haut et fort une formation continue dont la mise en pratique soit la plus libérale possible. Comment la SSMI gère-t-elle cette situation? 
On ne peut pas contenter tout le monde, c'est bien connu. La SSMI tient à définir une ligne de conduite bien claire, en se laissant guider par un objectif: il faut que, dans la mesure du possible, tous les internistes, aussi bien en cabinet médical qu'en milieu hospitalier, se perfectionnent et continuent à se former de manière permanente et optimale pour le bien des patients - selon des critères scientifiques, éthiques et économiques. Dans ce contexte, la SSMI met l'accent particulièrement sur la qualité des programmes de formation continue.

\section{Le Programme de formation continue de la SSMI}

Le contenu du Programme de formation continue de la SSMI est structuré de telle manière que les internistes, médecins hospitaliers et praticiens, puissent couvrir leurs «besoins médicaux fondamentaux» en matière de formation continue. Il s'agit en l'occurrence de médecine interne. Dans le Programme de formation continue, cette appellation englobe tout ce qui est nécessaire à un interniste «standard» pour pouvoir exercer son métier. Cela exclut donc certains domaines situés clairement en-dehors de la médecine interne (par exemple la médecine alternative), ainsi que les sujets administratifs ou d'autre nature (tel que le Tarmed).

La SSMI est d'avis qu'une société de discipline médicale est responsable de la formation continue dans les domaines scientifiques qui font partie de la spécialité de la société en question. En revanche, une société de discipline médicale n'est pas responsable de la formation continue pour l'ensemble des connaissances qu'un médecin doit posséder et mettre à jour régulièrement, par exemple en matière d'informatique ou d'assurances.

La manière dont on définit le besoin annuel en formation continue d'une spécialité reste un sujet controversé. Ce besoin est actuellement évalué à 50 heures par année (150 heures sur une période de trois ans), il constitue donc le minimum obligatoire que les médecins doivent effectuer, mais il est probable qu'en la matière, la solution idéale reste encore à trouver.

\section{Les manifestations de formation continue proposées par la SSMI}

Les sociétés de discipline médicale ont aussi la tâche essentielle de proposer des manifestations de formation continue. C'est un domaine dans lequel la SSMI est très active. Au cours de ces dernières années, le congrès annuel de la SSMI, qui avait tendance à être une manifestation quelque peu élitiste, un peu trop axée sur le monde hospitalier, est devenu une manifestation moderne, d'une grande actualité. Désormais, le congrès de la SSMI est le plus grand rassemblement médical de Suisse.

En plus du congrès annuel, la SSMI propose deux sortes de cours. Les cours qui se déroulent au niveau régional sont organisés directement par la SSMI; ils ont lieu principalement dans des cliniques de catégorie A (en 2003, à Langenthal, Lausanne et Neuchâtel). Ces cours rencontrent un succès qui va grandissant. Par ailleurs, la SSMI collabore à l'organisation de cours déjà établis, tels que le Cours de Davos, Quadrimed, le Fortbildungskurs der medizinischen Fakultät Zürich, le Zentralschweizer Internistenkurs et le Lenker Kurs. Un membre de la Commission pour la formation continue ou du Comité directeur collabore à l'organisation de ce deuxième type de cours.

Par ailleurs, sous le label de «SGIM goes regional», la SSMI a élaboré un plan cadre pour une nouvelle forme de cours, d'une demi-journée, dont le but est de mieux implanter la SSMI au niveau régional.

\section{Evaluation et homologation des manifes- tations de formation continue}

La SSMI procède à l'évaluation des divers programmes de formation continue. Dans ce contexte, elle ne se contente pas de porter un jugement sur le contenu, qui selon le Programme de formation continue doit être consacré à la médecine interne, mais elle examine également l'ensemble de la manifestation et les influences extérieures que son contenu pourrait subir (par exemple de la part de l'industrie pharmaceutique). Cette évaluation représente souvent un processus très complexe, voici pourquoi:

- Les manifestations doivent être jugées sur la base du programme remis à la SSMI, et non en fonction d'une impression personnelle que l'on se fait en tant que participant.

- Dans son appréciation, la SSMI doit tenir compte de la nature spécifique des différents types de programmes, qu'il s'agisse de E-Learning, de l'activité d'enseignant, de la rédaction de travaux scientifiques ou de la formation continue au sein des cercles de qualité etc.

- Peu de manifestations de formation continue peuvent encore se dérouler sans qu'elles aient bénéficié d'une collaboration entre les sociétés de discipline médicale et l'industrie pharmaceutique - c'est une réalité que l'on ne 
peut ignorer. C'est pourquoi il est particulièrement difficile et délicat de juger des manifestations sponsorisées.

Dans l'évaluation des manifestations de formation continue et dans l'attribution de «crédits», la SSMI applique des critères qui sont constamment affinés. L'expérience a montré que la plupart des organisateurs s'efforcent d'obtenir une validation de la part de la SSMI; les critères de qualité de la SSMI peuvent donc influencer les organisateurs de manière positive.

Récemment, le site internet de la SSMI a mis en ligne une banque de données dans laquelle figurent des manifestations et des programmes de formation continue reconnus par la SSMI. Actuellement, cette liste comprend plus de 1000 éléments dont le nombre ne cesse de grandir.

\section{La SSMI participe au processus décisionnaire}

La SSMI s'efforce de prendre part aux débats et aux prises de décisions des instances supérieures (législateur, FMH etc.). La SSMI y défend le point de vue que la fonction de la Réglementation pour la formation continue est d'être au service d'une médecine de grande qualité, libérale, et que ceci n'implique aucune autre restriction.

\section{Le contrôle de la formation continue est conforme à l'esprit du temps}

L'aspect obligatoire de la formation continue est fortement controversé. Ceux qui s'y opposent avancent souvent des arguments du genre:

- «Tout ça ne mène à rien.»

- «Avant, les médecins continuaient aussi à se perfectionner.»

- «Les 〈tire-au-flanc trouveront toujours un moyen de se dérober.»

- «Quand on voit tous les auditeurs assoupis et les <participants> qui ne se montrent qu'au moment où l'on distribue les attestations, on se pose de sérieuses questions.»

- «Personne n'a encore réussi à démontrer qu'une formation continue obligatoire a pour effet d'améliorer la qualité de la médecine pratiquée et surtout la santé des patients.»

C'est peut-être vrai. Il n'en demeure pas moins que le problème est nettement plus complexe et compliqué que ça. L'opinion publique exige de plus en plus que les médecins puissent justifier d'une formation continue accomplie. Et elle ne se satisfait plus de l'affirmation des médecins qui lui assurent: «Nous faisons ce qui est nécessaire, faites-nous simplement confiance.» Le législateur, les organisations de défense des consommateurs et des patients, les compagnies d'assurances, tous exigent que la formation continue soit contrôlée, car ils appliquent le fameux principe selon lequel «il est bien de faire confiance, mais il vaut tout de même mieux vérifier.» De la part de la population et des autorités on observe une nette tendance à exiger une plus grande transparence et à se montrer critique. C'est un phénomène qui ne concerne pas que les médecins, mais également d'autres catégories professionnelles.

\section{La SSMI et le contrôle de la formation continue}

Face à sa responsabilité d'assurer la mise en pratique de la formation continue, la SSMI a adopté une attitude ferme et logique: puisque le contrôle de la formation continue est obligatoire, elle ne peut tolérer que celui-ci ne dégénère en mascarade. Cela ne signifie nullement que la SSMI, se complaisant dans la dévotion et l'obéissance aveugle, courbe l'échine devant le législateur et les assureurs, mais simplement qu'elle ne tient pas à ce que les vérifications par sondage deviennent un simulacre vide de sens.

L'expérience a montré que la majorité des médecins accomplissent leur formation continue consciencieusement et avec un grand sens des responsabilités. Mais la SSMI a également constaté que le nombre de participants aux diverses manifestations de formation continue a augmenté de manière spectaculaire depuis que le perfectionnement est devenu obligatoire. Elle constate également une nette augmentation du nombre de programmes proposés aux médecins. On est donc en droit de se demander où tous ces «nouveaux» participants effectuaient leur formation continue dans le passé. En se basant sur leurs propres observations, les personnes en charge de la formation continue au sein de la SSMI pensent qu'il est avéré que depuis que la formation continue a été rendue obligatoire et vérifiable, la qualité des programmes et des manifestations s'est améliorée.

Pour une société de discipline médicale, il est souvent difficile de concilier les revendications, souvent justifiées, de ses membres et les exigences émanant des autorités et du législateur. La SSMI s'efforce de trouver des solutions judicieuses en matière de formation continue. Ses responsables sont conscients du fait qu'en tant que plus grande société de discipline médicale suisse, la SSMI assume aussi le rôle de premier de cordée dans cette difficile ascension. 